\title{
DETERMINAÇÃO DO FATOR DE CONTRAÇÃO PARA OS AÇOS PERITÉTICOS*
}

\author{
Afrânio Márcio Costa \\ Carlos Antônio da Silva² \\ Carlos Vinícius Domingos de Carvalho ${ }^{3}$ \\ Eduardo Teixeira de Oliveira Rocha ${ }^{4}$ \\ Itavahn Alves da Silva ${ }^{5}$ \\ Werner Aredes Matos ${ }^{6}$
}

\section{Resumo}

Placas lingotadas continuamente, não obstante atendam a requisitos como composição química, conteúdo de inclusões, aspectos macro e micrográficos, podem ser desclassificadas por falta de conformidade dimensional. Contrações térmicas, de solidificação e de transformações de fases são partes intrínsecas do processo e são afetadas por alterações na taxa de extração de energia da placa durante o processo. Este conjunto de contrações determina o Fator de Contração de operação, o qual é utilizado para determinar as dimensões do molde que irão resultar na largura especificada, onde o presente estudo determinará o fator de contração dos aços peritéticos. As variáveis identificadas que influenciam no fluxo de calor primário foram: frequência de oscilação, superaquecimento, velocidade de lingotamento e viscosidade do pó fluxante. Identificou-se que o resfriamento secundário e a conicidade utilizado no molde, também são critérios determinantes para obtenção de larguras especificadas. Assim sendo, uma equação de regressão foi desenvolvida para o Fator de Contração.

Palavras-chave: Fator de contração; Fluxo de calor; Pó fluxante.

\section{DETERMIANTION OF SHRINKAGE FACTOR FOR PERITECTIC STEEL GRADE}

\begin{abstract}
Continuously cast slabs, regardless of achieving requirements such as chemical composition, inclusion content, and macro and micrographic aspects, can be downgraded due to lack of dimensional conformity. Slab contraction due to thermal effects, solidification and phase transformations are intrinsic parts of the process and define the value of the Shrinkage Factor, which is used to evaluate the mold dimensions required to achieve the required slab size, where the present study determines the shrinkage factor of peritetic steel grade. The variables identified in the primary heat flux were: frequency oscillation, super heat, casting speed, viscosity of the casting powder. The secondary heat flux and the taper were also found to be important criteria to obtain the specified width. Therefore a regression equation was developed for the Shrinkage Factor.
\end{abstract}

Keywords: Shrinkage factor; Heat flux; Casting powder.

\footnotetext{
Engenheiro Metalurgista, Consultor Técnico de Aciaria- Gerdau.

Engenheiro Metalurgista, M.Sc.; Ph.D. na Universidade Federal de Ouro Preto.

Engenheiro Metalurgista, Assessor Técnico de Aciaria- Gerdau.

Engenheiro Mecânico, Chefe do Acabamento de Placas- Gerdau.

Engenheiro Metalurgista, M.Sc; D.Sc. na Universidade Federal de Ouro Preto.

Engenheiro Metalurgista, Graduado pela Universidade Federal de Ouro Preto.
} 


\section{INTRODUÇÃO}

O Fator de Contração é uma variável utilizada pelas empresas para ajustes e trocas de largura no molde durante o lingotamento. Neste caso, os ajustes realizados no molde buscam compensar a contração dos aços durante o processo de lingotamento a fim de obter placas dentro da largura especificada a frio. Em função da necessidade de buscar redução de custos com placas fora da tolerância largura, fezse necessário estudar um método que reduza a variabilidade de largura no Lingotamento Contínuo de Placas, sendo assim, tornou-se importante identificar quais os parâmetros que influenciam no fator de contração para os diferentes tipos de aços produzidos, por se tratar do principal parâmetro de influência na largura.

Velocidades de lingotamento abaixo ou acima do especificado, sem 0 correspondente ajuste de largura no molde, podem levar à extração de calor inadequada, podendo acarretar em placas com dimensional fora do especificado. Como mostra a Figuras 1 (a) e (b).

(a)

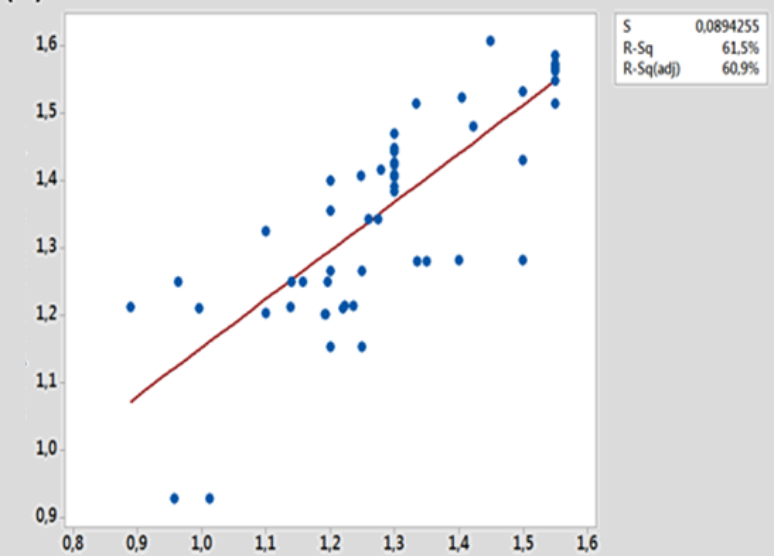

(b)

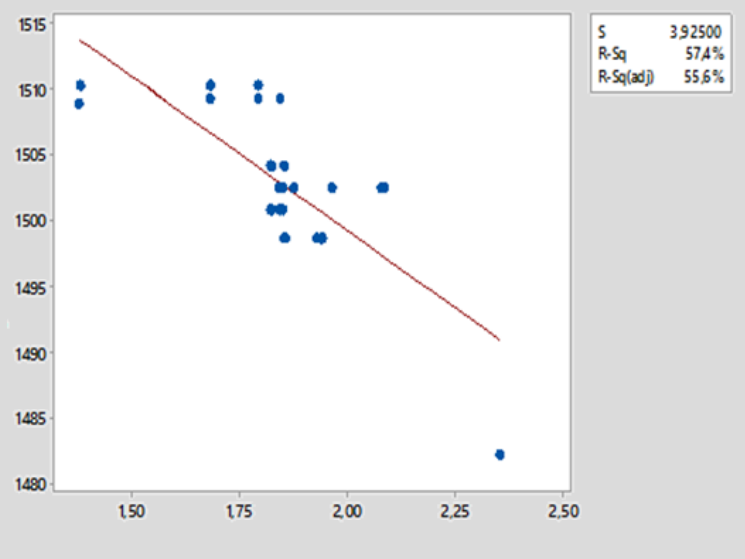

Figura 1. Variação do fluxo de calor de acordo com a velocidade (a). Variação de largura de acordo com o fluxo de calor (b).

O presente estudo buscou identificar quais parâmetros operacionais e metalúrgicos que são responsáveis pela variação de extração de calor, e sua influência sobre o grau de contração para o aço estudado. Esta informação, expressada por meio do Fator de Contração, permite ajustar a largura do molde de forma a conservar a qualidade dimensional das placas lingotadas para que os alargamentos de bobinas possam ser conhecidos. A Figura 2 mostra como o fator de contração é utilizado para dimensionar o molde. 


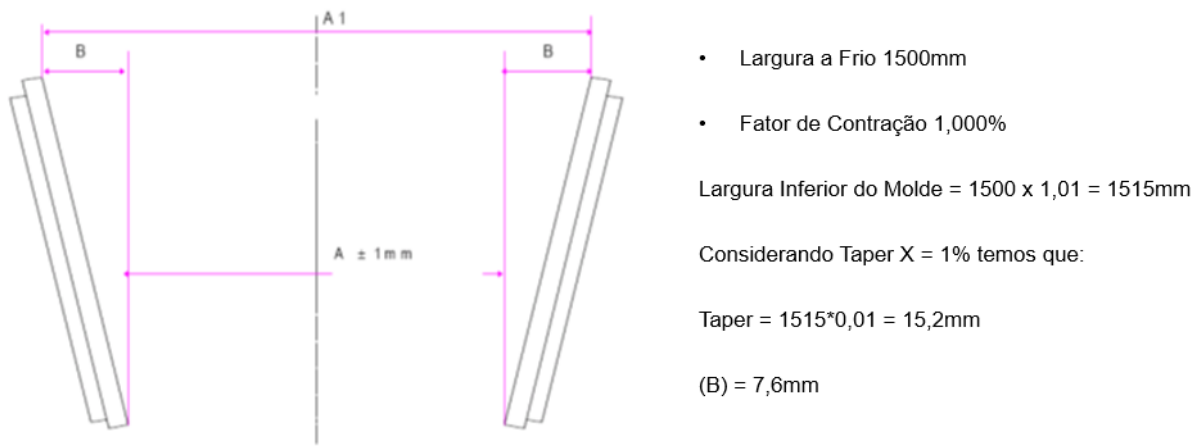

Figura 2. Dimensionamento do molde com base no fator de contração e na conicidade.

O fluxo de calor instantâneo é obtido como uma função do tempo de residência do aço no molde e, variáveis que influenciam a extração de calor do resfriamento primário. A extração de calor em um molde de lingotamento contínuo de placas ocorre de acordo com a Equação 1 [2].

$$
Q=4,63 \times 10^{6} \mu^{-0,09} T_{\text {fusão }}^{-1,19} V^{0,47}\left\{1-0,152 \exp \left[-\left(\frac{0,107-\% C}{0,027}\right)^{2}\right]\right\}
$$

Q Fluxo de calor $\left(\mathrm{MW} / \mathrm{m}^{2}\right)$;

$\mu$ Viscosidade do pó fluxante $\left(1300^{\circ} \mathrm{C}\right.$; Pa-s)

Tfusão Temperatura de fusão do pó fluxante $\left({ }^{\circ} \mathrm{C}\right)$;

$\checkmark$ Velocidade de lingotamento $(\mathrm{m} / \mathrm{min})$;

$\% \mathrm{C}$ porcentagem do carbono no aço.

Para altas velocidades, o tempo de solidificação é menor e a pele de solidificação é menos espessa, sendo portanto, empurrada mais facilmente pela pressão ferrostática contra a parede do molde como pode ser observado na Figura 3 (a) [2] e (b) [3].
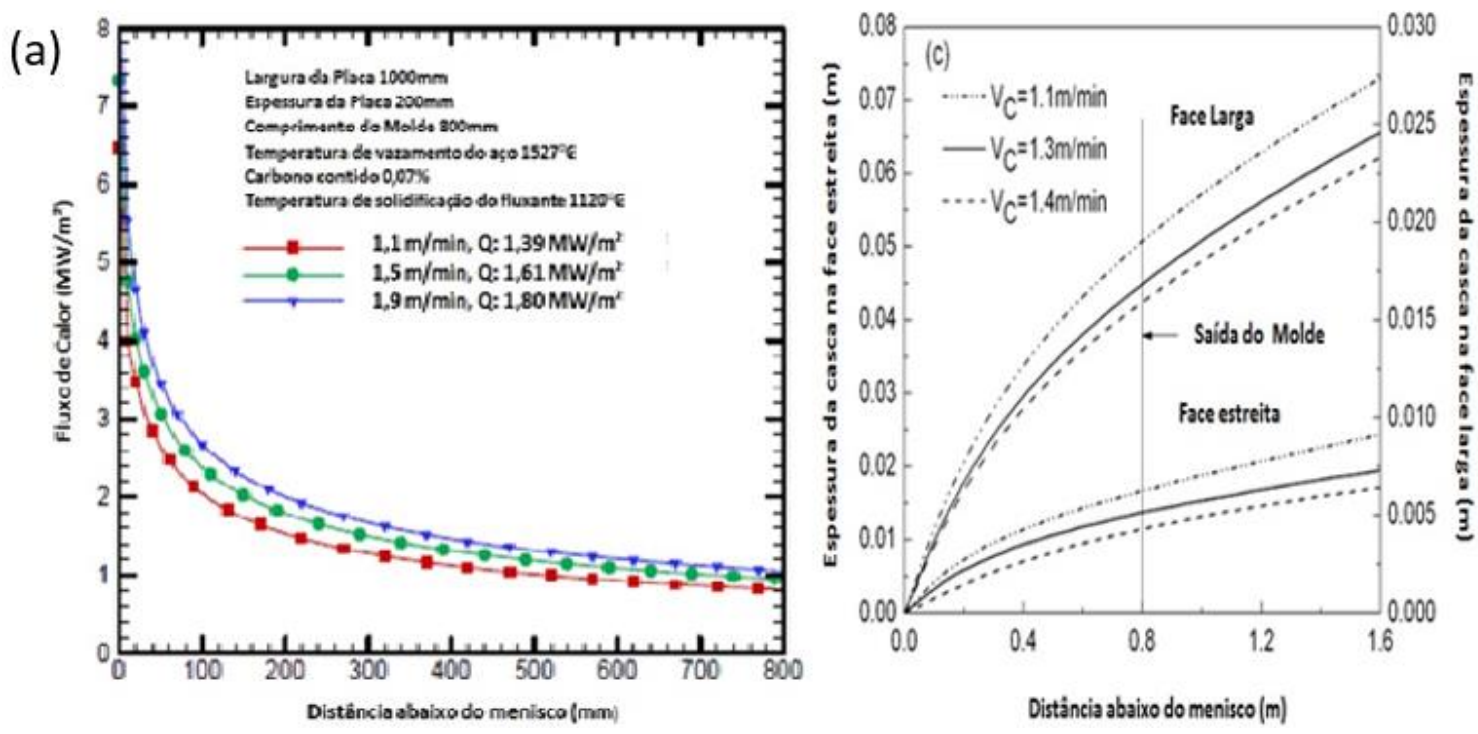

Figura 3. (a) variação do fluxo de calor de acordo com a velocidade, (b) espessura de pele de acordo com a velocidade de lingotamento. 
Sendo assim, os parâmetros que regem o fluxo de calor no molde serão de suma importância para quantificar o fluxo de calor nesta região.

Sob o ponto de vista térmico, o superaquecimento (super heat) tem um efeito reduzido no fluxo de calor, quando comparado as variáveis mais importantes como: velocidade de extração e tipo de fluxante [1].

A influência da frequência de oscilação durante o processo de lingotamento para aços peritéticos foi estudada, com base neste estudo ficou claro que a temperatura do molde aumentou com o aumento da frequência de oscilação e a transferência de calor também se comportou da mesma forma, ou seja, quanto mais alta a frequência, maior a transferência de calor [4].

As características do pó fluxante influenciam sobre a extração de calor no molde; para este fim, uma das principais características é a viscosidade de sua escória. No estudo em questão, foram utilizados fluxantes de viscosidade 0,07 e 0,08 Pa-s (a $1300^{\circ} \mathrm{C}$ ) para os aços das classes peritéticas. O fluxo de calor no molde tende a aumentar de acordo com a diminuição da viscosidade do pó fluxante e a temperatura de fusão da escória [5]. Sugere-se também que o fluxo de calor no molde varia de acordo com a viscosidade do pó fluxante, como mostra a Figura 4 $[6,7]$.

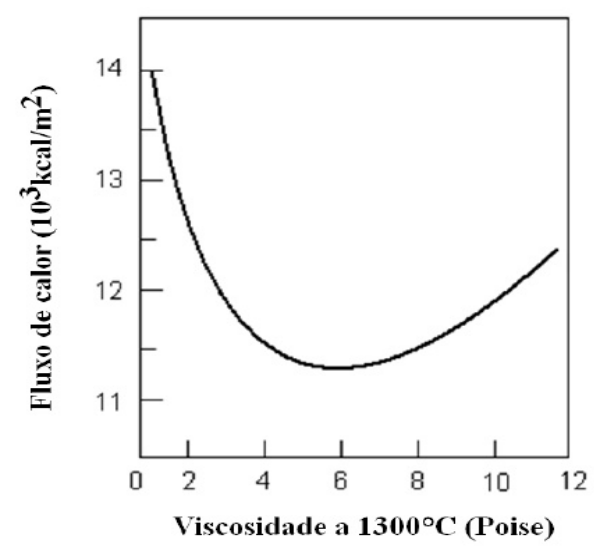

Figura 4 - Fluxo de calor no molde em função da viscosidade da escória do fluxante a uma

velocidade de lingotamento de $1,0 \mathrm{~m} / \mathrm{min}$.

Durante o tempo de estripamento negativo, a camada de pó fluxante líquida consegue penetrar na interface metal/molde, permitindo assim, uma melhor lubrificação dessa região e proporcionando um aumento na extração de calor [6,7].

A conicidade ideal deveria ser determinada de acordo com a curva de extração de calor no molde devido à diferença de extração de calor que se tem na região do menisco e na saída do molde [2].

A máquina em estudo possui sistema de resfriamento secundário com sprays mistos, esses borrifam uma mistura de água e ar nas placas. Neste caso, os coeficientes de transferência de calor são calculados pelas Equações 2 [8] e 3 [1]. Obteve-se assim uma extração de calor média para o resfriamento secundário de $0,63 \mathrm{MW} / \mathrm{m}^{2}$, onde a extração de calor média para o resfriamento primário é 1,3 $\mathrm{MW} / \mathrm{m}^{2}$. Sendo assim a contribuição do resfriamento primário ou fluxo de calor no molde é mais importante para a contração dos aços do que o resfriamento secundário, já que o resfriamento secundário tem como principal função controlar a 
temperatura de superfície das placas para reduzir a probabilidade de defeitos superficiais e internos.

$$
h=20 \times n \times(Q)^{0,55}
$$

h $\quad$ Coeficiente de transferência de calor $\left(\mathrm{W} / \mathrm{m}^{2} \mathrm{~K}\right)$;

n Número de sprays presente na zona de resfriamento;

Q Fluxo de água nas zonas de sprays $\left(\mathrm{L} / \mathrm{m}^{2} \mathrm{~s}\right)$.

$$
h r=\varepsilon \times \sigma\left(T s^{2}-T a g u a^{2}\right) \times(T s+T a g u a)
$$

$\mathrm{hr} \quad$ Coeficiente de transferência de calor por radiação $\left(\mathrm{W} / \mathrm{m}^{2} \mathrm{~K}\right)$;

$\varepsilon \quad$ Emissividade;

$\sigma \quad$ Constante de Stefan-Boltzmann;

Ts Temperatura da superfície da placa $(\mathrm{K})$;

Tágua Temperatura da água (K).

\section{MATERIAIS E MÉTODOS}

\subsection{Metodologia}

O Fator de Contração é influenciado pelas variáveis que afetam a taxa de extração de calor no molde. Espera-se, portanto, que os principais parâmetros a serem observados sejam a velocidade de lingotamento, frequência de oscilação, conicidade, superaquecimento, viscosidade do pó fluxante. De maneira a determinar a influência destes parâmetros, foram coletados dados que incluem a variação destes parâmetros em ao menos dois níveis. Os dados foram coletados para aços do tipo peritético com carbono entre $0,10 \%$ e $0,12 \%$ de composições químicas, que são da prática usual da Gerdau.

As placas produzidas sob determinada combinação dos parâmetros operacionais já citados, foram identificadas para determinação do Fator de Contração. Posteriormente, foi realizada uma medição de largura tanto na face $A$ (face superior) quanto na face $B$ (face inferior) à $400 \mathrm{~mm}$ da base e do topo da placa lingotada a frio, Figura 5, o que permitiu comparar a largura que se obteve a frio e a largura determinada na base do molde. Essas medidas foram utilizadas para a determinação do Fator de Contração dos aços estudados.

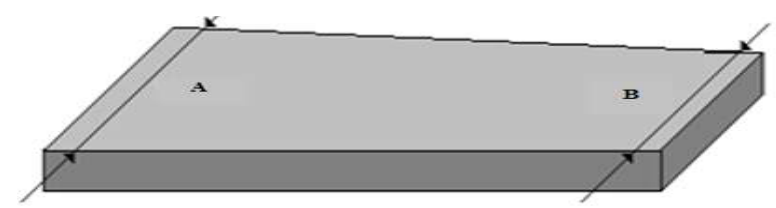

Figura 5. Regiões da placa que onde foram realizadas as medições.

\section{RESULTADOS E DISCUSSÃO}

Nesta seção, serão apresentadas as regressões obtidas a partir dos dados coletados buscando entender a influência metalúrgica de cada variável nas equações. Os dados de placas também serão apresentados nesta etapa mostrando 
como a determinação do Fator de Contração foi importante para conseguir obter faixas de especificação de larguras mais estreitas.

\subsection{Regressões para o Fluxo de Calor no Molde}

Foi realizada uma regressão do tipo stepwise, a fim de se determinar a influência de alguns parâmetros na extração de calor no molde.

A Figura 6 mostra que, assim como apresentado pela Equação 1 (3), a variável velocidade de lingotamento e viscosidade do pó fluxante têm influência direta na extração de calor do molde conforme já apresentado na Equação 1 [2]. Além disso, para a máquina em estudo, a frequência de oscilação e o superheat ou superaquecimento, também são responsáveis pelo fluxo de calor no molde.

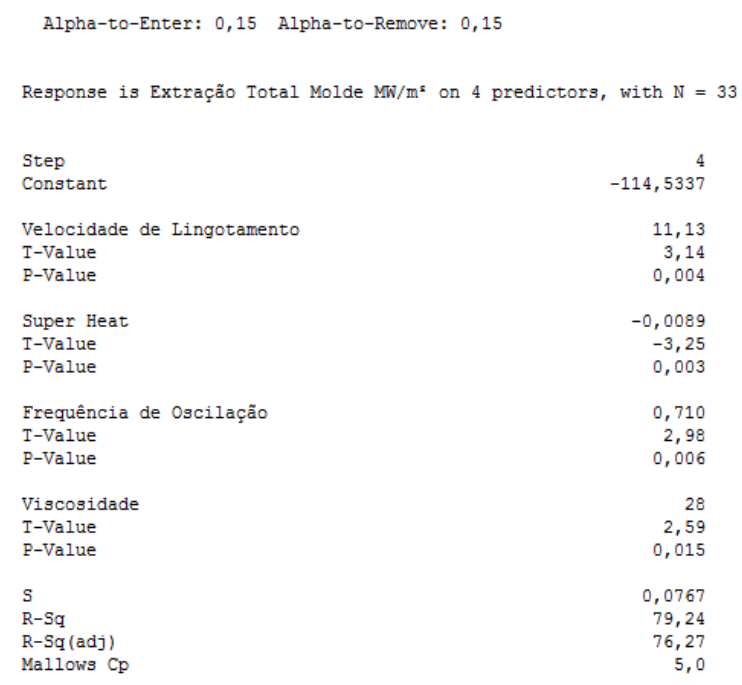

Figura 6. Regressão Stepwise para o fluxo de calor no molde para larguras de 800 a $1200 \mathrm{~mm}$.

A equação 4 correspondente a este ajuste, é referente aos aços com porcentagem de carbono de $0,10 \%$ a $0,12 \%$, que se enquadram na classe dos peritéticos.

$$
\mathrm{Q}=-115+11,1 \times \mathrm{V}-0,00887 \times \mathrm{SH}+0,710 \times \mathrm{f}-28,4 \times \mu(4)
$$

Q Fluxo de Calor $\left(\mathrm{MW} / \mathrm{m}^{2}\right)$;

$\mathrm{V} \quad$ velocidade de lingotamento $(\mathrm{m} / \mathrm{min})$;

$f \quad$ Frequência de Oscilação (Hertz);

$\mu \quad$ Viscosidade do Pó Fluxante $\left(1300^{\circ} \mathrm{C} \mathrm{Pa-s}\right)$;

$\mathrm{SH}$ Super Heat $\left({ }^{\circ} \mathrm{C}\right)$.

Espera-se que para os moldes, o aumento da velocidade de extração da placa leve a um aumento significativo do fluxo de calor. Tal fato se justifica: para altas velocidades, o tempo de residência no molde é menor; sendo assim, a pele mais fina é empurrada mais facilmente pela pressão ferrostática contra as paredes do molde, aumentando assim o fluxo de calor [1,2]. 
A Figura 7 apresenta um gráfico com a relação entre o fluxo de calor extraído no molde fornecido pelo sistema da máquina e os valores calculados pela regressão obtida acima.

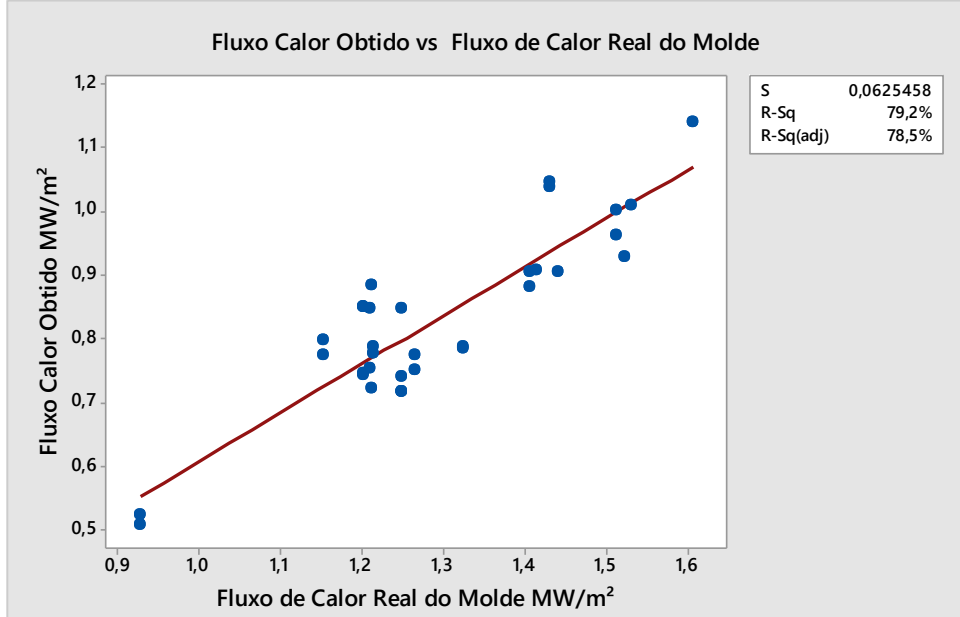

Figura 7. Fluxo de Calor real vs Fluxo de Calor obtido pela regressão para larguras 800 a $1200 \mathrm{~mm}$.

\subsection{Fator de Contração}

O Fator de Contração é uma razão entre a largura da base do molde e a largura da placa lingotada obtida a frio. No presente estudo foi feita a medição a frio das placas na banca de inspeção, e a largura da base do molde foi obtida através do sistema interno da máquina, obtendo-se assim os Fatores de Contração para cada placa.

A Figura 8 apresenta, para o caso deste estudo, as variáveis responsáveis por determinar o valor do Fator de Contração. Como se podem notar, as variáveis velocidade de lingotamento, viscosidade do pó $\left(a 1300^{\circ} \mathrm{C}\right)$, conicidade e superaquecimento (super heat), que são responsáveis pelo fluxo de calor na região primária, aparecem na equação do Fator de Contração. Tal fato faz com que a variável fluxo de calor primário não apareça explícita na equação de regressão por estar dissociada nestes parâmetros que a compõe.

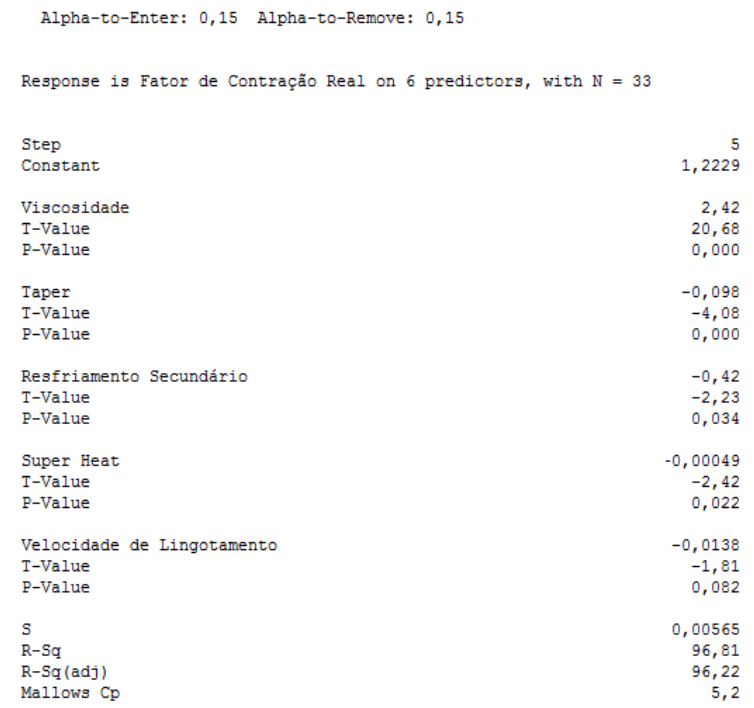

Figura 8. Regressão Stepwise para o Fator de Contração para larguras de 800 a 1200 mm. 
A equação 5 correspondente a este ajuste é referente aos aços com porcentagem de carbono de $0,10 \%$ a $0,12 \%$, que se enquadram na classe dos peritéticos.

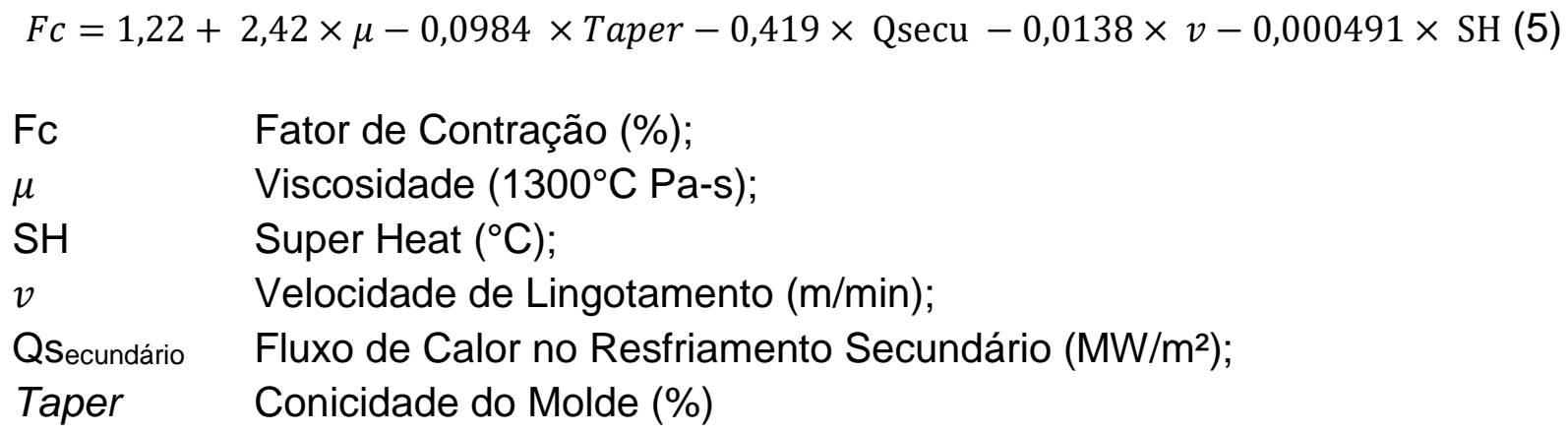

A Figura 9 apresenta um gráfico que relaciona o fator de contração real com o fator de contração obtido por meio da regressão.

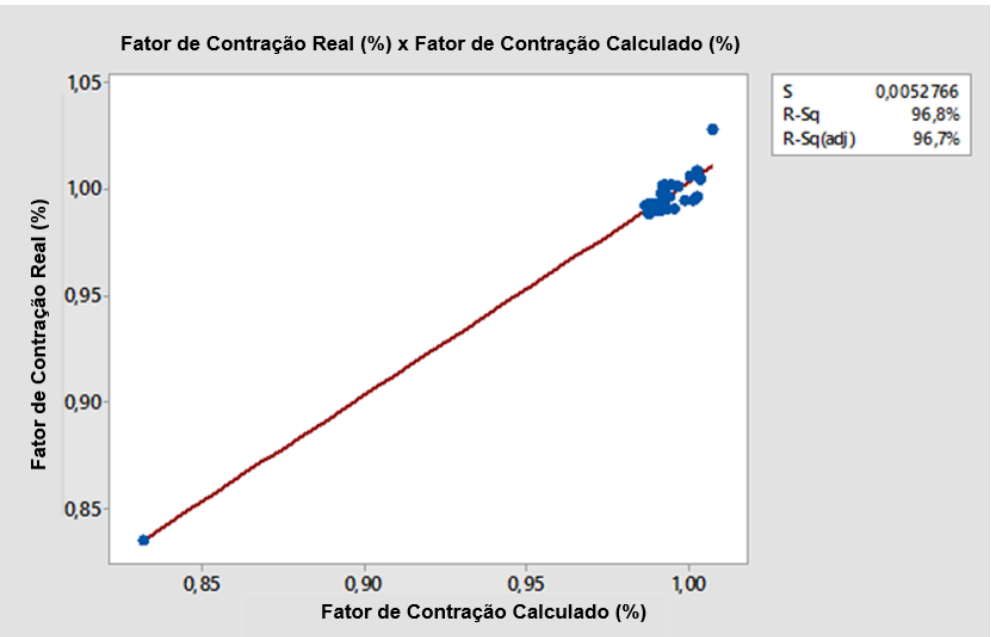

Figura 9 - Fator de Contração Real vs Fator de Contração calculado para aços com larguras entre 800 a $1200 \mathrm{~mm}$.

\subsection{Análise do Ajuste do Fator de Contração}

Ao determinar a fórmula do Fator de Contração, foi possível analisar e comparar os valores do Fator de Contração que eram utilizados antes na máquina de lingotamento contínuo de placas da Gerdau com o atual, como pode ser observado na Figura 10 (a) e (b).

(a) Largura média Geral- Fator de contração médio de 1,010- Peritético

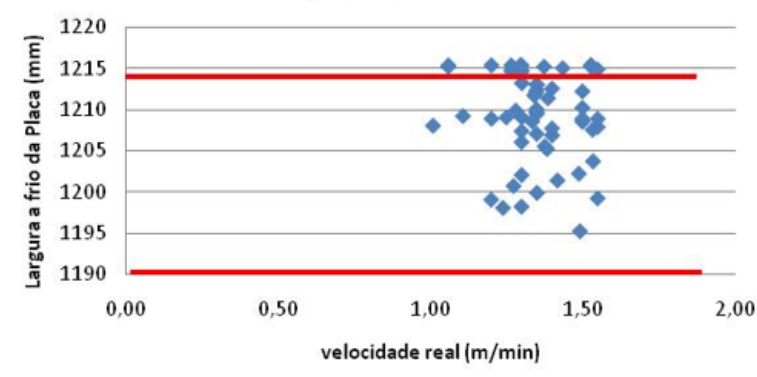

(b)

Largura média Geral- Fator de contração médio de 1,000. Peritético

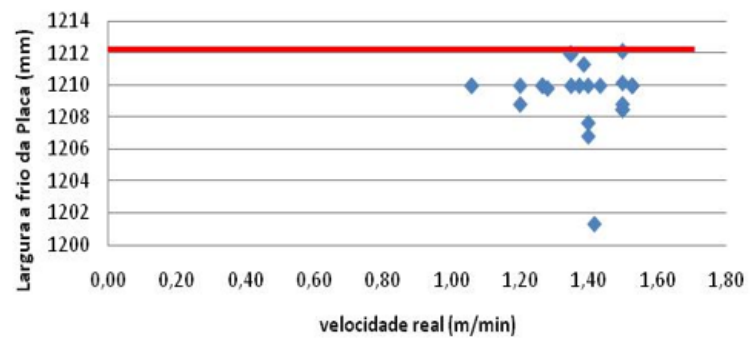

Figura 10. (a) Variação de largura a frio para Fc 1,010. (b) Variação de lagura a frio para Fc 1,000 
Nota-se que para Fc 1,010 algumas larguras de placas estão ultrapassando o limite de largura especificada, o que poderá acarretar bobinas com alargamento em excesso. Já para o Fc 1,000 todas as placas produzidas estão dentro do limite de largura especificado, o que leva a produção de bobinas dentro do especificado.

As Figuras 11 (a) e (b) mostram os histogramas com os limites de tolerância de (+/$15 \mathrm{~mm}$ ) de largura para o fator de 1,010 e a redução obtida de variação de largura de $(+/-12 \mathrm{~mm}$ ) para o de fator de 1,000, o que conferiu uma redução nos limites de especificação da largura de $6 \mathrm{~mm}$ o que garante a produção de Bobinas dentro do especificado.
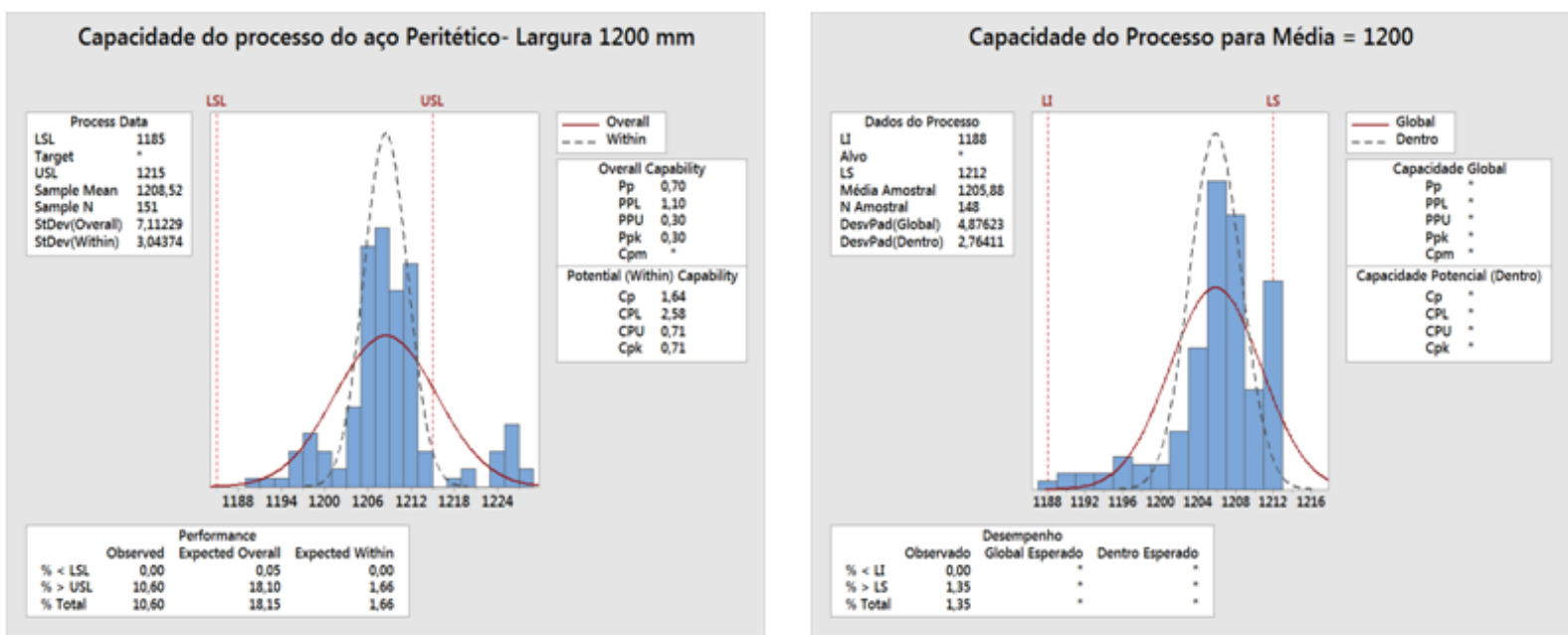

Figura 11. (a) Capacidade do processo do aço Peritético para larguras de placas com $1200 \mathrm{~mm}$ limites de tolerância de (+/- $15 \mathrm{~mm})$ de largura. (b) Capacidade do processo do aço Peritético para as larguras de placas com 1200mm limites de tolerância de (+/- $12 \mathrm{~mm})$ de largura

\section{CONCLUSÃo}

O estudo realizado mostrou que o fluxo de calor no molde, ou fluxo de calor primário, é de suma importância para obtenção de larguras dentro de especificado, onde os parâmetros responsáveis são: frequência de oscilação; superaquecimento (super heat); velocidade de lingotamento e viscosidade do pó fluxante.

Identificou-se que o fluxo de calor no resfriamento secundário e conicidade (taper) utilizado no molde, são também critérios importantes para obtenção de larguras dentro do especificado, porém, o fluxo de calor no molde ou também conhecido como extração de calor no resfriamento primário mostrou ser o parâmetro de maior importância para o fator de contração, sendo composta pelos seguintes parâmetros: velocidade de lingotamento; superaquecimento e viscosidade do pó fluxante. Assim sendo, uma equação de regressão foi desenvolvida para o Fator de Contração, tendo como variáveis dependentes: calor extraído no resfriamento secundário; superaquecimento (super heat); conicidade (taper); velocidade de lingotamento e viscosidade do pó fluxante;

O fator de contração foi alterado de 1,010 para 1,000 o que conferiu uma redução nos limites de especificação da largura de $6 \mathrm{~mm}$ o que garante a produção de Bobinas dentro do especificado. Este trabalho será estendido para outros tipos de aços e larguras. 


\section{REFERÊNCIAS}

1 VALADARES, C. A. G. Lingotamento Contínuo de Placas. São Paulo: Associação Brasileira de Metalurgia e Materiais (ABM). 2010. 13 p.

2 THOMAS, B.G.; OJEDA, C. Ideal Taper Prediction for Slab Casting 2003 ISSTech Steelmaking Conference, Indianapolis, IN, USA, April 27-30, 2003, ISS-AIME, Warrendale, PA, p. 295-308. 2003.

3 WANG, H.; LI. G.; LEI. Y.; ZHAO. Y.; DAI. Q.; WANG1'. J.; Mathematical Heat Transfer Model Research for the Improvement of Continuous Casting Slab Temperature, ISIJ International, v.45, pp 1291-1296, 2005.

4 PINHEIRO, C.A.; SAMARASEKERA, I.V.; BRIMACOMBE, J.K. \& WALKER, B.N. "Mould heat transfer and continuously cast billet quality with mould flux lubrification Part 1: mould heat transfer". Ironmaking Steelmaking, v. 27, n. 1, p. 37-53, 2000.

5 ALBERNY, R. et alli. La Lingotiére de Coulée Continue de Brames et sou Bilan Thermique C.I. T No $11-1976$.

6 Wolf M.M. Initial Solidification and Strand Surface Quality of Peritectic Steels, Vol. 9, Iron and Steel Society, Warrendale, PA, 1997, 1-111.

7 Wolf M.M. A Review of Published Work on the Solidification control of Steel in Contiunuous Casting Molds by Heat Flux Meassurement, ISIJ Internaticonal, v.20, pp 710-720, 1980.

8 F. RAMSTORFER; J. ROLAND; C. CHIMANI; K. MÖRWALD. Modelling of air-mist spray cooling heat transfer for continuous slab casting. International Journal of Cast Metals Research. v. 22, p 39-42, $2009 .$. 